\title{
A new colorectal cancer risk prediction model incorporating family history, personal and environmental factors
}

Yingye Zheng, ${ }^{1,2}$ Xinwei Hua, ${ }^{1,3}$ Aung K. Win, ${ }^{4,5}$ Robert J. MacInnis, ${ }^{4,6}$ Steven

Gallinger, ${ }^{7}$ Loic Le Marchand, ${ }^{8}$ Noralane M. Lindor, ${ }^{9}$ John A. Baron, ${ }^{10}$ John L. Hopper ${ }^{4}$ James G. Dowty, ${ }^{4}$ Antonis C. Antoniou, ${ }^{11}$ Jiayin Zheng, ${ }^{1}$ Mark A. Jenkins, ${ }^{* 4}$ Polly A. Newcomb*\#1,3

${ }^{1}$ Division of Public Health Sciences, Fred Hutchinson Cancer Research Center, Seattle, Washington, USA

${ }^{2}$ Department of Biostatistics, University of Washington School of Public Health, Seattle, Washington, USA

${ }^{3}$ Department of Epidemiology, University of Washington School of Public Health, Seattle, Washington, USA

${ }^{4}$ Centre for Epidemiology and Biostatistics, Melbourne School of Population and Global Health, The University of Melbourne, Parkville, Victoria, Australia

${ }^{5}$ Genetic Medicine, Royal Melbourne Hospital, Parkville, Victoria, Australia

${ }^{6}$ Cancer Epidemiology Centre, Cancer Council Victoria, Melbourne, Victoria, Australia

${ }^{7}$ Lunenfeld Tanenbaum Research Institute, Mount Sinai Hospital, University of Toronto, Toronto, Ontario, Canada 
${ }^{8}$ Epidemiology Program, University of Hawaii Cancer Center, Honolulu, Hawaii, USA

${ }^{9}$ Department of Health Science Research, Mayo Clinic Arizona, Scottsdale, Arizona, USA

${ }^{10}$ Department of Medicine University of North Carolina School of Medicine, and

Department of Epidemiology, Gillings School of Global Public Health Chapel Hill, North

Carolina, USA

${ }^{11}$ Cancer Research UK, Genetic Epidemiology Unit, Department of Public Health and

Primary Care, University of Cambridge, Cambridge, UK

* Joint last author

\# For correspondence

For correspondence:

Polly A Newcomb, PhD, MPH

Fred Hutchinson Cancer Research Center

1100 Fairview Avenue N., M4-B402

Seattle, WA 98109

Phone: 206-667-3476

Fax: 206.667.7056

Email: pnewcomb@fredhutch.org 
Running title: Colorectal cancer risk prediction

Keywords: Colorectal cancer, family history, risk prediction, screening

Presented at AACR special conference "Improving Cancer Risk Prediction for Prevention and Early Detection" 2016, "Does a comprehensive family history of colorectal cancer improve risk prediction?"

Funding: This work was supported by (R01 CA170122) from the National Cancer Institute, National Institutes of Health $(\mathrm{NIH})$ and through cooperative agreements with the following Colon Cancer Family Registry (CCFR) centers: Australasian Colorectal Cancer Family Registry (U01/U24 CA097735), Mayo Clinic Cooperative Family Registry for Colon Cancer Studies (U01/U24 CA074800), Ontario Familial Colorectal Cancer Registry (U01/U24 CA074783), Seattle Colorectal Cancer Family Registry (U01/U24 CA074794), and USC Consortium Colorectal Cancer Family Registry (U01/U24 CA074799).

Seattle CCFR research was also supported by the Cancer Surveillance System of the Fred Hutchinson Cancer Research Center, which was funded by Control Nos. N01-CN-67009 and N01-PC-35142 and Contract No. HHSN2612013000121 from the Surveillance, Epidemiology and End Results (SEER) Program of the National Cancer Institute. Additional support included grants from the National Institutes of Health UM1/U01 CA167551, K05 CA152715 (to PAN) and R01 GM085047 (to YZ), and through the Centre for Research Excellence grant APP1042021 and Program Grant 
APP1074383 from the National Health and Medical Research Council (NHMRC),

Australia. MAJ is a NHMRC Senior Research Fellow. AKW is a NHMRC Early Career

Fellow. JLH is a NHMRC Senior Principal Research Fellow.

Notes: The funders had no role in the design of the study; the collection, analysis, and interpretation of the data; the writing of the manuscript; and the decision to submit the manuscript for publication.

The authors have no conflicts of interest to disclose. 


\section{Abstract}

Purpose: Reducing colorectal cancer (CRC) incidence and mortality through early detection would improve efficacy if targeted. A CRC risk-prediction model incorporating personal, family, genetic and environmental risk factors could enhance prediction.

Methods: We developed risk-prediction models using population-based CRC cases $(\mathrm{N}=4,445)$ and controls $(\mathrm{N}=3,967)$ recruited by the Colon Cancer Family Registry Cohort (CCFRC). A familial risk profile (FRP) was calculated to summarize individuals' risk based on their CRC family history, family structure, germline mutation probability in major susceptibility genes, and a polygenic component. Using logistic regression, we developed risk models including individuals' FRP or a binary CRC family-history (FH), and risk factors collected at recruitment. Model validation used follow-up data for population- $(\mathrm{N}=12,052)$ and clinic-based $(\mathrm{N}=5,584)$ relatives with no cancer history at recruitment, assessing calibration (E/O) and discrimination (AUC).

Results: The E/O (95\% confidence interval [CI]) for FRP models for population-based relatives were $1.04(0.74-1.45)$ and $0.86(0.64-1.20)$ for men and women, and for clinicbased relatives $1.15(0.87-1.58)$ and $1.04(0.76-1.45)$. The age-adjusted AUC $(95 \% \mathrm{Cl})$ for FRP models in population-based relatives were $0.69(0.60-0.78)$ and $0.70(0.62-$ $0.77)$, and for clinic-based relatives 0.77 (0.69-0.84) and 0.68 (0.60-0.76). The incremental values of AUC $(95 \% \mathrm{Cl})$ for $\mathrm{FRP}$ over $\mathrm{FH}$ models for population-based relatives were $0.08(0.01-0.15)$ and $0.10(0.04-0.16)$, and for clinic-based relatives 0.11 (0.05-0.17) and $0.11(0.06-0.17)$.

Conclusion: The FRP-based model and FH-based model calibrate well in both settings. The FRP-based model provided better risk-prediction and discrimination than 
bioRxiv preprint doi: https://doi.org/10.1101/662106; this version posted June 7, 2019. The copyright holder for this preprint (which was not certified by peer review) is the author/funder, who has granted bioRxiv a license to display the preprint in perpetuity. It is made available under aCC-BY-ND 4.0 International license.

the FH-based model. A detailed family history may be useful for targeted risk-based screening and clinical management. 


\section{INTRODUCTION}

Screening for colorectal cancer (CRC) is efficient and cost effective. ${ }^{1}$ The evidence is compelling, even when applied irrespective of personal characteristics except age. ${ }^{2}$ However, beyond age, individuals' CRC risk factors could inform the use and frequency of specific screening regimens. ${ }^{3,4}$ A detailed risk prediction model would permit targeted screening at an appropriate level based on individuals' risk of $\mathrm{CRC}$. $^{5-8}$

Family history of CRC is an important risk factor for this disease, as it is a proxy for genetic and environmental factors shared by relatives. ${ }^{9,10}$ A comprehensive risk prediction model would incorporate detailed family history of cancer and available information on known genetic, and epidemiologic characteristics. To date, existing CRC or colorectal adenoma risk prediction models are limited, ${ }^{7}$ including simple measures of CRC family history and limited risk factor data, ${ }^{11-16}$ or considering only a small group of known low-penetrance SNPs that explain little familial aggregation of CRC. ${ }^{17}$ Moreover, of the seven models that have been validated using external samples, they were found to have only reasonable discrimination, suggesting limited usefulness for risk-based screening. ${ }^{18}$

The majority of these risk models defined family history as a binary variable, typically as "at least one first-degree relative with CRC"; a few models considered family history as the number of first- or second-degree relatives with CRC. A small number of models (e.g., MMRpro) ${ }^{19}$ used a more complex definition based on the number of affected relatives, their ages of $\mathrm{CRC}$ diagnosis and the degree of relatedness. In theory, the more detailed and accurate the family history, the better the risk prediction. However, in a typical primary care setting with limited time and incomplete patient 
reports, only the presence or absence of a CRC family history is generally recorded. It is unclear whether such information is sufficient to predict CRC risk accurately. Using the large well-characterized population-based data from the Colon Cancer Family Registry Cohort (CCFRC), ${ }^{20,21}$ we describe the development and validation of a new risk prediction model that incorporates a novel measure of family history in addition to personal and environmental risk factors.

\section{METHODS}

\section{Study sample}

The CCFRC is an NCl-funded international consortium of six $\mathrm{CRC}$ registries from the USA, Canada, and Australia/New Zealand, using standard protocols for data collection, molecular characterization, and follow-up at each site (http://coloncfr.org/). Recently diagnosed CRC cases from population-based cancer registries, controls from population-based sources (including drivers' license, voting records, health beneficiary rosters, and electoral rolls), and cases from family cancer clinics with a strong family history of CRC or early-onset disease were recruited as "probands" between 1998$2012 .^{20}$ Relatives of population- and clinic-based cases were also invited to participate. Informed consent was obtained from participants in all study sites. Local institutional research ethics review boards approved the study protocols. 


\section{Data collection and testing}

At baseline, participants were asked to: (i) complete an epidemiological risk factor questionnaire on medical history, demographic characteristics, reproductive history, physical activity, medication, postmenopausal hormone use, alcohol and tobacco use, and diet about one year before diagnosis or a comparable period in controls; (ii) describe detailed CRC family history information, at least for their first degree relatives, including relationship to the participant, age, sex, and type and ages of cancer diagnosis; (iii) provide written consent for the research team to access tumor tissues and corresponding pathological reports; and (iv) collect a blood or buccal sample. Reported cancers and ages at diagnosis were confirmed, where possible, using pathology reports, medical records, cancer registry reports and/or death certificates. Genetic mutation screening and testing for mismatch repair (MMR) and MUTYH mutations was completed for probands and relatives, as previously described in detail. ${ }^{22}$ During follow-up approximately every 5 years, participants from all case families were contacted for updates on incident polyps, cancer diagnoses at any site, CRC screening and surgery, as well as among their relatives cancer diagnoses and deaths. Populationbased controls were not followed up.

\section{Family history measures}

Family history of CRC was defined in two ways: 1) as a binary indicator (yes/no) of having at least one first-degree relative with $\mathrm{CRC}$ (hereafter called, $\mathrm{FH}$ ); and 2) as a continuous familial risk profile (FRP) based on detailed cancer family history, considering age at disease diagnosis as well as the number of and relationship to each 
relative, their ages, and their probabilities of carrying $\mathrm{CRC}$ predisposing mutations in the DNA MMR genes (MLH1, MSH2, MSH6, PMS2) and MUTYH genes. ${ }^{22,23}$ The FRP can be considered as a probability index, indicating absolute risk of $\mathrm{CRC}$ from birth to age 80 years. The FRP was calculated based on modified segregation analysis using: (i) the age- and sex-specific incidence of CRC from national cancer statistics; ${ }^{24}$ (ii) the familial relative risk based on previous segregation analysis of $\mathrm{CRC}$ data from the CCFR; ${ }^{22}$ and (iii) the age-specific incidence of CRC based on mutation status, for which we used the penetrance reported from analysis of the CCFRC (Supplementary Methods). ${ }^{22,25-29}$ We included established pre-diagnosis risk factors in model development, including screening (as described in Tables 1-2 and Supplementary Methods). A list of candidate variables and their parameterization used for model selection are described in Supplementary Table 1.

\section{Statistical analysis}

\section{Model development}

We studied 4,445 CRC cases and 3,967 controls recruited from three populationbased sites of the CCFRC (Seattle, WA, USA; Ontario, Canada; and Victoria, Australia). We only included cases who were diagnosed with CRC less than two years before completing the baseline questionnaire to ensure all risk factor pertained to the prediagnostic period. Controls were frequency-matched to the cases on age. We restricted our analysis to non-Hispanic whites. Participants with missing values on any of the baseline data variables were excluded from model development. 
The CRC risk prediction model was developed using unconditional logistic regression with case/control status as the outcome. The distributions of FRP by sex and case-control status were examined using histograms and compared using Wilcoxon non-parametric tests. Models using either log-transformed FRP or FH were stratified by sex to permit sex-specific associations with risk factors. We adjusted for study site and reference age (age at diagnosis of $\mathrm{CRC}$ for cases and age at interview for controls) in all models. To account for site-specific sampling of CCFRC, we applied probability weights based on the (inverse) sampling probability of each individual's selection into the study. ${ }^{20}$

Three forward-stepwise-selection procedures were implemented. Each used a different selection criteria: 1) $P$ value $<0.15$; 2) incremental value in Area Under the Receiver Operating Characteristic (ROC) Curve (incAUC) $\geq 0.01$; or 3 ) a smaller Akaike information criterion (AIC). ${ }^{30,31}$ Final models from these different selection criteria were compared to identify variables that were robust to selection procedures. For example, if having a colorectal polyp was included in the variable set in a model based on incAUC, and as well as that from a AIC-based selection procedure, then it would then be included as a predictor in the final model. Two definitions of family history (FH and logtransformed FRP) were included in our final models in addition to the final list of environmental factors.

\section{Relative and absolute risk calculation}

Odds ratios (ORs) and corresponding 95\% confidence intervals $(\mathrm{Cl})$ were generated from the final models. To calculate absolute risk, we obtained sex- and age- 
specific CRC incidences for the USA (SEER-9 Registries, whites), Australia (Victoria) and Canada (Ontario) populations from the Cancer Incidence in Five Continents (Cl5), for $1998-2002,{ }^{29}$ corresponding approximately to the time period during which cases were diagnosed. Deaths from non-CRC causes were considered as competing risks. Age- and sex-specific mortality from causes other than CRC were obtained from allcause mortality ${ }^{32-34}$ and CRC-specific mortality ${ }^{35}$ for the USA, Australia and Canada respectively during the same time period. Five-year absolute risks were calculated as described in Freedman et al. ${ }^{14}$ (Supplementary Methods).

\section{Model Validation}

For model validation, we studied 17,636 unaffected relatives of case probands who: (1) were recruited from population registries $(\mathrm{N}=12,052)$ and genetic clinics $(\mathrm{N}=5,584)$; (2) were non-Hispanic whites; (3) had no personal history of any cancer at the time of recruitment; (4) completed a baseline questionnaire; and (5) were recruited from five study sites of the CCFRC (Australia/New Zealand, Mayo Clinic, Ontario, Cedars-Sinai and Seattle) ${ }^{20}$ and (6) were prospectively followed up after the baseline recruitment. The flowchart of the study design and model steps is included as

\section{Supplementary Figure 1.}

We calculated absolute risk for each individual in the validation set based on our final model, the derived baseline risk functions, and data from the baseline questionnaire. In total, $12.6 \%$ of men and $17.0 \%$ of women had at least one covariate with missing data (see Supplementary Table 2). We conducted imputation for each risk factor variable in the final model with the most frequent (modal) category for men 
and women separately. ${ }^{36}$ Model performance was compared before and after exclusion of relatives with missing covariates (Supplementary Table 3). Using the follow-up data of the CCFRC, we identified incident CRC diagnoses. Time to event was defined as years from the date of baseline interview completion to the date of diagnosis of incident CRC. Individuals with a diagnosis of other types of cancer were censored at the date of diagnosis. Deaths due to causes other than CRC were considered as competing risk events. Participants who were alive without any cancer diagnosis were censored at the date of last contact.

Given different incidence rates for disease in the general population and the genetic clinic pool, we assessed model performance separately for population- and clinic- based relatives. For calibration performance, we categorized participants into quintiles of predicted absolute risk based on the developed model and plotted the average observed absolute risk within each quintile against the predicted risks in that quintile, adding 95\% Cls of the observed risks. The observed marginal risks were calculated as the cumulative incidences of CRC accounting for censoring and competing risks. ${ }^{37}$ In addition, we calculated a summary measure of calibration for the FRP and FH models as the ratio of the averaged predicted 5-year absolute risk to the observed cumulative incidence rate $(\mathrm{E} / \mathrm{O})$, separately for men and women; $95 \% \mathrm{Cls}$ were calculated using a bootstrap approach. ${ }^{38}$ To assess the performance of the model for risk stratification, we defined four risk-groups based on the $30^{\text {th }}, 60^{\text {th }}$ and $90^{\text {th }}$ percentiles of the predicted 5-year absolute risks of CRC. For each model, we plotted cumulative incidence functions of CRC diagnosis by risk-groups, as above, and tested differences among risk functions across groups using K-sample test. ${ }^{39}$ Since clinic- 
based population have higher $\mathrm{CRC}$ incidence rate than the rates from population-based registries, for clinic-based relatives, we calculated their baseline risk using the clinicbased set from our validation population.

For discrimination performance, we conducted Receiver Operating Characteristic (ROC) curve analyses and calculated AUC (Supplementary Methods) to assess the model's ability to separate individuals with and without a CRC diagnosis within 5 years after baseline. Since the outcome of individuals who were censored within 5 years after baseline was uncertain, we excluded these individuals in this calculation and used inverse censoring probability weights to account for the missing information. Two age groups were defined as $\leq 50$ and $>50$ years old at baseline separately for both men and women. Age-adjusted ROC curves and AUCs were calculated as the weighted average of age-specific AUC, with weights as the proportion of $\mathrm{CRC}$ diagnosis in each age group. We used a bootstrap approach to calculate the empirical $95 \% \mathrm{Cls}$ for ageadjusted AUC based on 2.5 and 97.5 percentiles. Analyses were conducted using $R$ version 3.1.1 (http://www.r-project.org/). All statistical tests were two-sided and $P$ values of less than 0.05 were considered statistically significant.

\section{Results}

\section{Model development}

Using population-based cases and controls, the variables entered into the final models of FRP and FH for men and women are shown in Tables $\mathbf{1}$ and 2, respectively. The distribution of FRP (range: 0.037 to 0.993 ) by sex and case-control status is 
summarized in Table 3 and Supplementary Figure 2. For both men and women, cases had higher FRP than controls (all $P<0.001$ ).

For men, every $10 \%$ relative increase in FRP (e.g. 0.33 vs 0.30 ) was associated with $16 \%$ higher risk of developing CRC (95\% Cl: $11 \%-20 \%)$. From the $\mathrm{FH}$ model, the OR for family history was $2.34(95 \% \mathrm{Cl}: 1.90,2.88)$. The strengths of association with the other variables were similar for FRP and FH models (Table 1). For women, a 10\% relative increase in FRP was associated with 9\% higher risk of $\mathrm{CRC}(95 \% \mathrm{Cl}: 6 \%-12 \%)$. From the FH model, the OR for family history was $1.72(95 \% \mathrm{Cl}: 1.39-2.12)$. The strengths of associations with other variables were essentially no different than those from the FRP model (Table 2).

\section{Model validation}

The median follow-up time was 8.6 years; 317 relatives were diagnosed with incident $\mathrm{CRC}$ during this period. Calibration for population- and clinic-based relatives across a wide range of risk groups is presented in Supplementary Figure 3. The overall E/O estimates $(95 \% \mathrm{Cl})$ for different models are summarized in Table 4 . For population- based relatives, FRP and $\mathrm{FH}$ models calibrated well, with E/O estimates $(95 \% \mathrm{Cl})$ of $1.0(0.7-1.4)$ and $0.9(0.6-1.2)$ for men and women from FRP models, and 0.9 (0.6-1.2) and 0.8 (0.6-1.2) from FH models. For clinic-based relatives, FRP and FH models calibrated well with $\mathrm{E} / \mathrm{O}$ ranging from 1.0 to 1.2 .

In addition, we defined four groups at different levels of predicted risks (using $30^{\text {th }}, 60^{\text {th }}, 90^{\text {th }}$ percentiles as cutoffs). The cumulative incidence curves are presented for population- and clinic-based relatives separately (Figure 1a and 1b). The wider 
separation of the FRP models suggests they performed better than the FH models in stratifying individuals into distinctive risk groups.

The FRP model also provided improved discriminatory capacity over the FH model (Figure 2). For population-based relatives, the age-adjusted AUCs for the FRP model was $0.73(95 \% \mathrm{Cl}=0.67-0.79)$ for men and $0.70(95 \% \mathrm{Cl}=0.62-0.77)$ for women. The increments in age-adjusted AUC (incAUC) for FRP over FH models were 0.08 $(95 \% \mathrm{Cl}=0.01-0.15)$ for men, and $0.10(95 \% \mathrm{Cl}=0.04-0.16)$ for women (both excluding the null with $P<0.001)$. For clinic-based relatives, the age-adjusted AUCs (95\%CI) for FRP models were $0.77(0.69-0.84)$ and $0.68(0.60-0.76)$ for men and women, respectively. The incAUC $(95 \% \mathrm{CI})$ for FRP over $\mathrm{FH}$ models was $0.11(0.05-0.17)$ for men and $0.11(0.06-0.17)$ for women.

\section{Discussion}

We developed and validated a new risk prediction model which incorporated detailed family history information captured by the FRP, as well as personal and environmental risk factors. Generally, both FRP and FH models provided good calibration, however, our results also suggested that the FRP-based model gave better discrimination than a model using a simple binary summary of family history. ${ }^{1}$

One clinical utility of $\mathrm{CRC}$ risk models is to provide information for screening regimens tailored to an individual's risk, and to inform intensity of screening, decisions regarding chemoprevention, and utilization of gene panel testing. Current $\mathrm{CRC}$ screening recommendations are based solely on age and simple measures of family

\footnotetext{
${ }^{1}$ This model is available online at http://crisptool.org/crisp-int
} 
history. ${ }^{40}$ Our study suggests that consideration of multiple risk factors, including a detailed family history of CRC, can lead to the identification of individuals across the spectrum of $\mathrm{CRC}$ risk, from those at very low risk with delayed and/or non-invasive screening recommendations, to those at high risk for whom earlier screening and more frequent/invasive monitoring is recommended. We have shown that family history of $\mathrm{CRC}$ is an important factor for $\mathrm{CRC}$ risk prediction, either defined as a binary (yes/no) measure or based on FRP calculated from the family structure, cancer histories, and MMR/MUTYH mutation status. Our research supports two approaches to risk prediction for CRC. In settings where family history information is limited, the risk model could include only the simple present/absent question. In settings such as genetic clinics where family history information is likely to be more complete, the risk model could make use of the FRP to derive more precise risk discrimination.

Numerous risk models have been developed to predict CRC and colorectal adenomas based on CRC family history, genetic mutation screening, personal characteristics, and known risk factors - singly or in combination. ${ }^{7,18}$ However, our FRPbased risk model is unique in its incorporation of all these CRC risk factors and in its use of our novel familial risk measure based on detailed family history information. Risk models that use family history as a binary indicator do not account for variability in family size, age, or structure, age of CRC diagnosis, or the relationship of affected relatives to the proband, which are integral to characterizing familial risk. ${ }^{9,23,41}$ Both models evaluated in our study included environmental factors, as have most prediction models, to take advantage of the substantial contribution of these exposures on CRC risk. ${ }^{42}$ 
Our study has many strengths, including its population-based design for the model development, and cases a broad spectrum of familial risk. All risk factors were collected by the CCFRC sites using the same instrument. In particular, the assessment of family structure and cancer history was extensive. ${ }^{23,41}$ Finally, the CCFRC's use of a prospective follow-up design provided a validation data set with the same wellannotated information and from the same cohort upon which the model was developed.

Our study had some limitations. Although the validation of the model was prospective with epidemiologic factors assessed at baseline interview, the development of the model was based on retrospective reports of lifestyle and environmental exposures prior to recruitment. Additionally, since our cohort started over a decade ago, information on $\mathrm{CRC}$ screening might not reflect the most current screening practices, with newer screening tests and intervals now recommended. ${ }^{40}$ Further, since the absolute risks were derived based on the age-, sex-, and country- specific incidence from the general population, our model is well calibrated to population-based samples. We recalibrated the baseline risks for the clinical-specific model, using the clinic-based subset from our validation dataset. Future studies are needed to independently evaluate the calibration of this model especially for high-risk families. In addition, susceptibility SNPs identified by recent GWAS should be included to enhance the FRP-based model. $^{43,44}$

In conclusion, we developed and validated a new CRC risk prediction model that incorporates a novel measure of family history, the FRP, in addition to personal characteristics and other non-family history-based risk factors. The new FRP-based model provided better risk discrimination than the $\mathrm{FH}$-based model, suggesting that 
bioRxiv preprint doi: https://doi.org/10.1101/662106; this version posted June 7, 2019. The copyright holder for this preprint (which was not certified by peer review) is the author/funder, who has granted bioRxiv a license to display the preprint in perpetuity. It is made available under aCC-BY-ND 4.0 International license.

more detailed family history has the potential to be more informative for risk-based clinical decision-making.

Acknowledgements: The authors thank all study participants of the Colon Cancer Family Registry and staff for their many contributions to this project. 


\section{References}

1. Doubeni CA. Precision Screening for Colorectal Cancer: Promise and Challenges. Ann Intern Med. 2015.

2. Slattery ML, Edwards SL, Ma KN, et al. Colon cancer screening, lifestyle, and risk of colon cancer. Cancer Causes \& Control. 2000;11(6):555-63.

3. Haggar FA, Boushey RP. Colorectal cancer epidemiology: incidence, mortality, survival, and risk factors. Clin Colon Rectal Surg. 2000;22(4):191-7.

4. Potter JD. Risk factors for colon neoplasia--epidemiology and biology. Eur $J$ Cancer. 1995;31A(7-8):1033-8.

5. Freedman AN, Seminara D, Gail MH, et al. Cancer risk prediction models: a workshop on development, evaluation, and application. J Natl Cancer Inst. 2005;97(10):715-23.

6. Zauber AG, Lansdorp-Vogelaar I, Knudsen AB, et al. Evaluating test strategies for colorectal cancer screening: a decision analysis for the U.S. Preventive Services Task Force. Ann Intern Med. 2008;149(9):659-69.

7. Win AK, Macinnis RJ, Hopper JL, et al. Risk prediction models for colorectal cancer: a review. Cancer Epidemiol Biomarkers Prev. 2012;21(3):398-410.

8. Taplin SH, Ichikawa L, Buist DS, et al. Evaluating organized breast cancer screening implementation: the prevention of late-stage disease? Cancer Epidemiol Biomarkers Prev. 2004;13(2):225-34.

9. Taylor DP, Burt RW, Williams MS, et al. Population-based family history-specific risks for colorectal cancer: a constellation approach. Gastroenterology. 2010;138(3):877-85.

10. Henrikson NB, Webber EM, Goddard KA, et al. Family history and the natural history of colorectal cancer: systematic review. Genet Med. 2015;17(9):702-12.

11. Colditz GA, Atwood KA, Emmons K, et al: Harvard report on cancer prevention volume 4: Harvard Cancer Risk Index. Risk Index Working Group, Harvard Center for Cancer Prevention. Cancer Causes \& Control 11:477-88, 2000.

12. Imperiale TF, Wagner DR, Lin CY, et al. Risk of advanced proximal neoplasms in asymptomatic adults according to the distal colorectal findings. $N$ Engl $J$ Med. 2000;343(3):16974.

13. Driver JA, Gaziano JM, Gelber RP, et al. Development of a risk score for colorectal cancer in men. Am J Med. 2007;120(3):257-63.

14. Freedman AN, Slattery ML, Ballard-Barbash R, et al. Colorectal cancer risk prediction tool for white men and women without known susceptibility. J Clin Oncol. 2009;27(5):686-93. 
15. Wei EK, Colditz GA, Giovannucci EL, et al. Cumulative risk of colon cancer up to age 70 years by risk factor status using data from the Nurses' Health Study. Am J Epidemiol. 2009;170(7):863-72.

16. Ma E, Sasazuki S, Iwasaki M, et al. 10-Year risk of colorectal cancer: development and validation of a prediction model in middle-aged Japanese men. Cancer Epidemiol. 2010;34(5):534-41.

17. Jenkins MA, Makalic E, Dowty JG, et al. Quantifying the utility of single nucleotide polymorphisms to guide colorectal cancer screening. Future Oncol. 2016;12(4):50313.

18. Usher-Smith JA, Walter FM, Emery JD, et al. Risk Prediction Models for Colorectal Cancer: A Systematic Review. Cancer Prev Res (Phila). 2016;9(1):13-26.

19. Chen S, Wang W, Lee $\mathrm{S}$, et al. Prediction of germline mutations and cancer risk in the Lynch syndrome. JAMA. 2006;296(12):1479-87.

20. Newcomb PA, Baron J, Cotterchio M, et al. Colon Cancer Family Registry: an international resource for studies of the genetic epidemiology of colon cancer. Cancer Epidemiol Biomarkers Prev. 2007;16(11):2331-43.

21. Jenkins MA, Win AK, Templeton AS, et al. Cohort Profile: The Colon Cancer Family Registry Cohort (CCFRC). International Journal of Epidemiology: 2018;47(2):387-388i.

22. Win AK, Jenkins MA, Dowty JG, et al. Prevalence and Penetrance of Major Genes and Polygenes for Colorectal Cancer. Cancer Epidemiol Biomarkers Prev. 2017;26(3):404-412.

23. Hopper JL. Disease-specific prospective family study cohorts enriched for familial risk. Epidemiol Perspect Innov. 2011;8(1):2.

24. Cancer incidence in five continents, Vol. IX. IARC Sci Publ. 2008;1-837.

25. Dowty JG, Win AK, Buchanan DD, et al. Cancer risks for MLH1 and MSH2 mutation carriers. Hum Mutat. 2013;34(3):490-7.

26. Baglietto L, Lindor NM, Dowty JG, et al. Risks of Lynch syndrome cancers for MSH6 mutation carriers. J Natl Cancer Inst. 2010;102(3):193-201.

27. Senter L, Clendenning M, Sotamaa K, et al. The clinical phenotype of Lynch syndrome due to germ-line PMS2 mutations. Gastroenterology. 2008;135(2):419-28.

28. Win AK, Dowty JG, Cleary SP, et al. Risk of colorectal cancer for carriers of mutations in MUTYH, with and without a family history of cancer. Gastroenterology. 2014;146(5):1208-11 e1-5.

29. Forman D, Bray F, Brewster DH, et al. Cancer Incidence in Five Continents, in Cancer IAfRo (ed), 2014. 
30. Akaike $\mathrm{H}$. Information Theory and an Extension of the Maximum Likelihood Principle. In Parzen E, Tanabe K, Kitagawa G (eds): Selected Papers of Hirotugu Akaike. New York, NY: Springer New York;1998:199-213.

31. Bozdogan H. Model selection and Akaike's Information Criterion (AIC): The general theory and its analytical extensions. Psychometrika. 1987;52(3):345-370.

32. Statistics Canada. Table 102-0504 - Deaths and mortality rates, by age group and sex, Canada, provinces and territories, annual, CANSIM (database).

33. Australian Institute of Health and Welfare General Record of Incidence of Mortality books 2013: All causes combined, 2015.

34. Deaths: Final Data for 2000, National Vital Statistics Reports 2002.

35. World Health Organization, health statistics and information systems, cancer mortality database.

36. Harrell F. Regression modeling strategies with applications to linear models, logistic and ordinal regression, and survival analysis. $2^{\text {nd }}$ ed. Switzerland: Springer; 2015.

37. Uno H, Cai T, Pencina MJ, et al. On the C-statistics for evaluating overall adequacy of risk prediction procedures with censored survival data. Stat Med. $2011 ; 30(10): 1105-17$.

38. Tibshirani RJ, Efron B. An introduction to the bootstrap. Monographs on statistics and applied probability 57. $1^{\text {st }}$ ed. New York: Chapman \& Hall; 1993.

39. Gray RJ. A Class of K-Sample Tests for Comparing the Cumulative Incidence of a Competing Risk. Annals of Statistics. 1988;16(3):1141-1154.

40. Levin B, Lieberman DA, McFarland B, et al: Screening and surveillance for the early detection of colorectal cancer and adenomatous polyps, 2008: a joint guideline from the American Cancer Society, the US Multi-Society Task Force on Colorectal Cancer, and the American College of Radiology. CA Cancer J Clin. 2008;58(3):130-160.

41. Yasui Y, Newcomb PA, Trentham-Dietz A, et al. Familial relative risk estimates for use in epidemiologic analyses. Am J Epidemiol. 2006;164(7):697-705.

42. WCRF/AICR: Food, Nutrition, Physical Activity, and the Prevention of Cancer: a Global Perspective. Washington DC, AICR, 2007.

43. Hsu L, Jeon J, Brenner $\mathrm{H}$, et al. A model to determine colorectal cancer risk using common genetic susceptibility loci. Gastroenterology. 2015;148(7):1330-9.e14.

44. Jeon J, Du M, Schoen RE, et al. Determining Risk of Colorectal Cancer and Starting Age of Screening Based on Lifestyle, Environmental, and Genetic Factors. Gastroenterology. 2018;154(8):2152-2164.e19. 
Table 1. Associations between risk factor variables and coloretal cancer from the risk model with familial risk profile (FRP model) and from the risk model with a binary family history (FH model), for men only

\begin{tabular}{|c|c|c|c|c|}
\hline \multirow{2}{*}{ Variables } & \multirow{2}{*}{$\begin{array}{c}\text { Cases }(\mathrm{N}=2312)^{*} \\
\text { No. (\%) }\end{array}$} & \multirow{2}{*}{$\begin{array}{c}\text { Controls }(\mathrm{N}=1916)^{\star} \\
\text { No. }(\%)\end{array}$} & \multirow{2}{*}{$\begin{array}{l}\text { FRP Model† } \\
\text { OR }(95 \% \mathrm{Cl})\end{array}$} & \multirow{2}{*}{$\begin{array}{l}\text { FH Mode I† } \\
\text { OR }(95 \% \mathrm{Cl})\end{array}$} \\
\hline & & & & \\
\hline \multicolumn{5}{|l|}{ Family History } \\
\hline FRP, Mean (SD) & $0.09(0.097)$ & $0.07(0.027)$ & $1.16(1.11,1.20)^{\natural}$ & -- \\
\hline \multicolumn{5}{|l|}{ Binary FH, No. (\%) } \\
\hline No & $1859(80.4)$ & $1731(90.3)$ & -- & 1.00 (Ref) \\
\hline Yes & $453(19.6)$ & $185(9.7)$ & & $2.34(1.90,2.88)$ \\
\hline \multicolumn{5}{|l|}{ Recent BMI ${ }^{\S}, \mathrm{kg} / \mathrm{m}^{2}$} \\
\hline$<25$ & $571(24.6)$ & $600(31.3)$ & 1.00 (Ref) & 1.00 (Ref) \\
\hline $25-30$ & $1113(48.0)$ & $940(49.1)$ & $1.38(1.18,1.62)$ & $1.35(1.15,1.58)$ \\
\hline$>30$ & $611(26.3)$ & $372(19.4)$ & $1.59(1.31,1.93)$ & $1.61(1.32,1.95)$ \\
\hline \multicolumn{5}{|c|}{ Red meat consumption, } \\
\hline \multicolumn{5}{|l|}{ servings/day } \\
\hline$<1$ & $1681(72.4)$ & $1484(77.5)$ & 1.00 (Ref) & 1.00 (Ref) \\
\hline
\end{tabular}


$1+$

Regular NSAID use duration",

years

Non-user

$\leq 2$

$>2$

Calcium supplement use duration,

years

Non-user

$\leq 2.5$

$>2.5$

Cigarette Smoking, pack-years

Never

$<10$

10 to 19
$564(24.3)$

$364(19.0)$

$1.27(1.08,1.51)$

$1.25(1.06,1.47)$

$1364(58.8)$

$475(20.5)$

$406(17.5)$

$2060(88.8)$

$115(5.0)$

$84(3.6)$

$810(34.9)$

$332(14.3)$

$289(12.5)$
$939(49.0) \quad 1.00$ (Ref) $\quad 1.00$ (Ref)

$388(20.3) \quad 0.91(0.76,1.09) \quad 0.93(0.78,1.10)$

$509(26.6) \quad 0.73(0.61,0.87) \quad 0.72(0.61,0.86)$

$\begin{array}{rcc}1610(84.0) & 1.00 \text { (Ref) } & 1.00 \text { (Ref) } \\ 97(5.1) & 1.29(0.95,1.75) & 1.32(0.97,1.79) \\ 137(7.2) & 0.62(0.46,0.83) & 0.62(0.46,0.83)\end{array}$

$689(36.0) \quad 1.00$ (Ref) $\quad 1.00$ (Ref)

$281(14.7) \quad 1.00(0.81,1.23) \quad 1.03(0.84,1.27)$

$229(12.0) \quad 1.18(0.95,1.47) \quad 1.16(0.93,1.45)$ 
$20+$

History of polypł

No

Yes

History of FOBT‡

No

Yes

History of sigmoidoscopy‡

No

Yes

History of colonoscopył

No

Yes
$807(34.8)$

2079 (89.6)

159 (6.9)

1569 (67.6)

$646(27.8)$

$1893(81.6)$

$330(14.2)$

2035 (87.7)

217 (9.3)
$632(33.0)$

$1.31(1.11,1.55)$

$1.34(1.14,1.58)$

$1691(88.3)$

1.00 (Ref)

1.00 (Ref)

$197(10.3)$

$1.46(1.10,1.95)$

$1.46(1.10,1.94)$

$1175(61.3)$

1.00 (Ref)

1.00 (Ref)

$667(34.8)$

$0.79(0.67,0.93)$

$0.80(0.68,0.95)$

BMI: Body mass index; FRP: Familial Risk Profile; FH: binary family history; FOBT: fecal occult blood test; NSAID:

Nonsteroidal anti-inflammatory drugs; $\mathrm{OR}=$ odds ratio; $\mathrm{Cl}=$ confidence interval. 
* Do not sum to total due to missing values; $\mathrm{N}$ for each category of variables unless otherwise specified.

${ }^{\dagger}$ Both models adjusted for study site and age at reference (age at diagnosis for cases and age at baseline interview for controls) in addition to the variables presented in this table.

${ }^{\ddagger}$ History of polyp, FOBT, sigmoidoscopy and colonoscopy were defined as history of having any of these conditions/test two years prior to enrollment.

$\S$ As of two years before enrollment.

${ }$ Regular NSAID use was defined as use of aspirin and/or ibuprofen at least twice a week for more than a month.

"OR for FRP: per 10\% increase. 
Table 2. Associations between risk factor variables and colorectal cancer from the risk model with familial risk profile (FRP model) and from the risk model with a binary family history (FH model), for women only

\begin{tabular}{|c|c|c|c|c|}
\hline Variables & $\begin{array}{c}\text { Cases }(\mathrm{N}=2133)^{\star} \\
\text { No. }(\%)\end{array}$ & $\begin{array}{c}\text { Controls }(\mathrm{N}=2051)^{\star} \\
\text { No. }(\%)\end{array}$ & $\begin{array}{l}\text { FRP Model † } \\
\text { OR }(95 \% \mathrm{Cl})\end{array}$ & $\begin{array}{l}\text { FH Model † } \\
\text { OR }(95 \% \mathrm{Cl})\end{array}$ \\
\hline \multicolumn{5}{|l|}{ Family History } \\
\hline FRP, Mean (SD) & $0.07(0.090)$ & $0.05(0.024)$ & $1.09(1.06,1.12)^{\|}$ & -- \\
\hline \multicolumn{5}{|c|}{ Binary FH, No. (\%) } \\
\hline No & $1708(80.1)$ & $1799(87.7)$ & -- & 1.00 (Ref) \\
\hline Yes & 425 (19.9) & 252 (12.3) & & $1.72(1.39,2.12)$ \\
\hline \multicolumn{5}{|l|}{ Recent BMI ${ }^{\S}, \mathrm{kg} / \mathrm{m}^{2}$} \\
\hline$<25$ & $971(45.5)$ & $1055(51.4)$ & 1.00 (Ref) & 1.00 (Ref) \\
\hline $25-30$ & $631(29.6)$ & $591(28.8)$ & $1.19(1.00,1.41)$ & $1.20(1.02,1.43)$ \\
\hline$>30$ & $501(23.5)$ & $380(18.5)$ & $1.40(1.15,1.70)$ & $1.42(1.17,1.72)$ \\
\hline \multicolumn{5}{|c|}{ Red meat consumption, } \\
\hline \multicolumn{5}{|l|}{ servings/d } \\
\hline$<1$ & $1721(80.7)$ & 1694 (82.6) & 1.00 (Ref) & 1.00 (Ref) \\
\hline
\end{tabular}


$1+$

Fruit consumption, servings/d

$<1$

$1+$

Smoking, pack-years

Never

$<10$

10 to 19

$20+$

Calcium use duration, years

Non-user

$\leq 2.5$

$>2.5$

History of polypł

No
$288(13.5)$

$228(11.1)$

$1.48(1.18,1.85)$

$1.47(1.17,1.85)$

$525(24.6)$

$1536(72.0)$

$1003(47.0)$

$392(18.4)$

$236(11.1)$

$411(19.3)$

$1268(59.4)$

$294(13.8)$

$378(17.7)$

$1943(91.1)$
$427(20.8)$

1.00 (Ref)

1.00 (Ref)

$1588(77.4) \quad 0.83(0.70,0.99)$

$0.83(0.69,0.99)$

$1047(51.0) \quad 1.00$ (Ref) $\quad 1.00$ (Ref)

$363(17.7) \quad 1.13(0.93,1.38) \quad 1.16(0.95,1.41)$

$174(8.5) \quad 1.13(0.88,1.45) \quad 1.13(0.88,1.45)$

$388(18.9) \quad 1.02(0.84,1.25) \quad 1.04(0.85,1.26)$

$985(48.0) \quad 1.00$ (Ref) $\quad 1.00$ (Ref)

$279(13.6) \quad 0.98(0.80,1.21) \quad 0.96(0.78,1.19)$

$564(27.5) \quad 0.81(0.67,0.97) \quad 0.80(0.67,0.96)$

$1835(89.5) \quad 1.00($ Ref $)$

1.00 (Ref) 
Yes

History of FOBT‡

No

Yes

History of sigmoidoscopy $\ddagger$

No

Yes

History of colonoscopy

No

Yes

Postmenopausal hormones

use

Non-user

Estrogen only

Estrogen + Progesterone
$129(6.0)$

177 (8.6)

$1.48(1.06,2.07)$

$1.50(1.07,2.09)$

$1502(70.4)$

$541(25.4)$

$1747(81.9)$

281 (13.2)

$1890(88.6)$

197 (9.2)

$1591(74.6)$

207 (9.7)

$126(5.9)$
$1635(79.7) \quad 1.00$ (Ref) $\quad 1.00$ (Ref)

$373(18.2) \quad 0.39(0.30,0.51)$

$373(18.2) \quad 0.39(0.30,0.51)$

$0.39(0.30,0.52)$

$1201(58.6)$

1.00 (Ref)

1.00 (Ref)

$793(38.7)$

$0.78(0.65,0.94)$

$0.77(0.64,0.93)$

$472(71.8) \quad 1.00$ (Ref) $\quad 1.00$ (Ref)

$502(24.5) \quad 0.68(0.56,0.84) \quad 0.68(0.55,0.84)$

$0.39(0.30,0.52)$

$1347(65.7)$

1.00 (Ref)

1.00 (Ref)

$277(13.5) \quad 1.03(0.82,1.31)$

$1.05(0.83,1.33)$

$183(8.9) \quad 0.81(0.61,1.07)$

$0.82(0.62,1.08)$ 
only

Mixed

$96(4.5)$

$145(7.1)$

$0.92(0.67,1.27)$

$0.90(0.66,1.24)$

BMI: Body mass index; FRP: Familial Risk Profile; FH: binary family history; FOBT: fecal occult blood test; OR= odds ratio; $\mathrm{Cl}=$ confidence interval.

* Do not sum to total due to missing values; $\mathrm{N}$ for each categories of variables unless otherwise specified.

† Both models adjusted for study site and age at reference (age at diagnosis for cases and age at baseline interview for controls) in addition to the variables presented in this table.

‡ History of polyp, FOBT, sigmoidoscopy and colonoscopy were defined as history of having any of these conditions/test two years prior to enrollment.

$\S$ As of two years before enrollment

"OR for FRP: per 10\% increase. 
Table 3. Distribution of Familial Risk Profile (FRP) by sex and case-control status

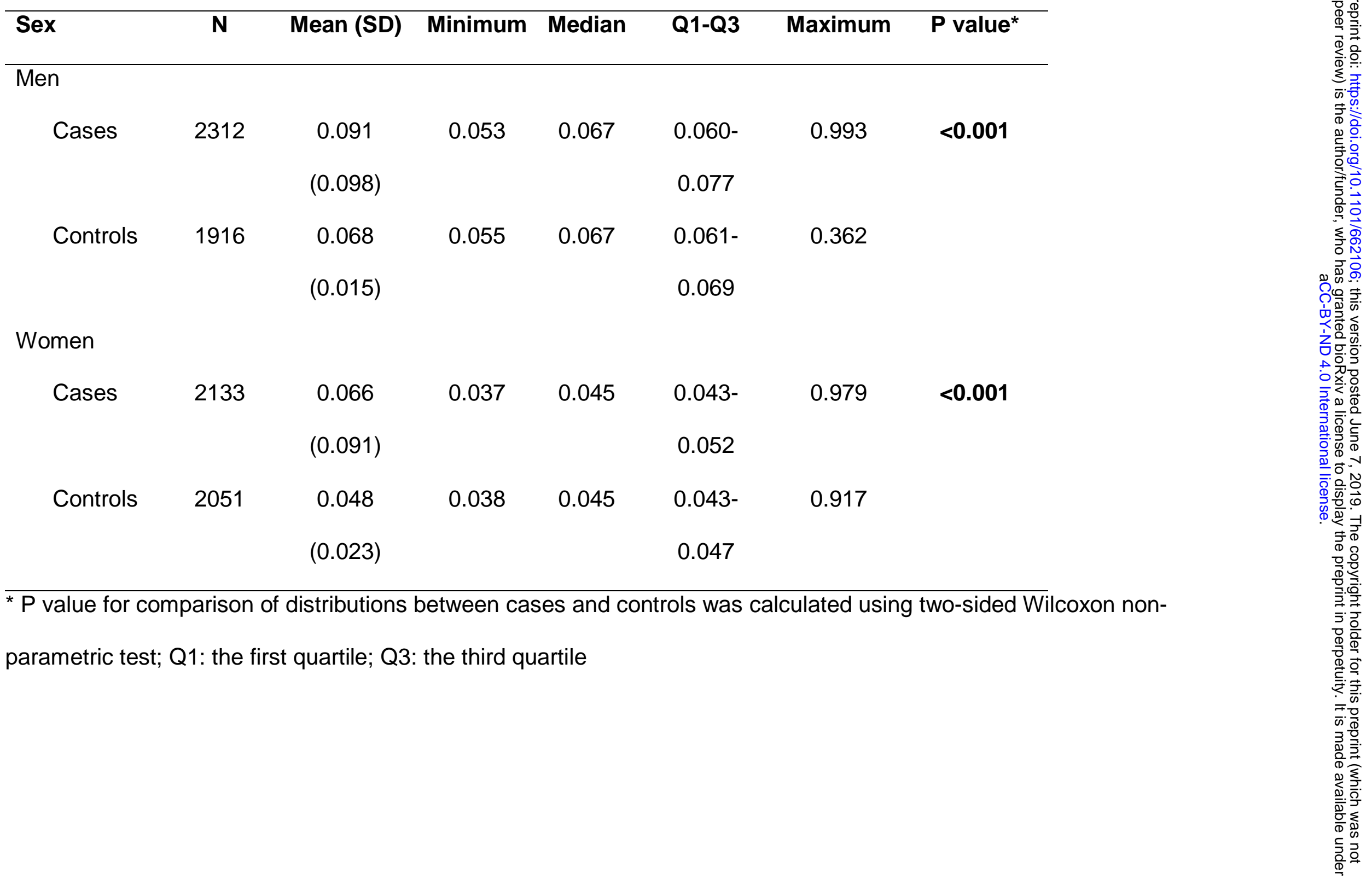


Table 4. Observed 5-year cumulative incidence rates (0) versus Averaged 5-year absolute risk $(E)$ based on risk models with familial risk profile or with a binary family history, and separately for men and women*

Sex/ Model

$$
\begin{gathered}
\text { Expected averaged 5- } \\
\text { year absolute risk (per } \\
1000 \text { person-years) } \\
(95 \% \mathrm{Cl})
\end{gathered}
$$

\section{Observed 5-year}

cumulative

incidence rates (per $\quad E / O(95 \% \mathrm{Cl})$

1000 person year)

$(95 \% \mathrm{Cl})$

\section{Population-based relatives}

Men

FRP model

$7.1(6.7-7.5)$

$7.1(4.9-9.6)$

$1.04(0.74-1.45)$

$\mathrm{FH}$ model

$6.1(5.9-6.3)$

$0.88(0.63-1.23)$

Women

FRP model

$5.2(5.0-5.5)$

FH model

$5.2(5.0-5.4)$

$6.3(4.4-8.2)$

\section{Clinic-based relatives}

Men

FRP model

$21.2(19.9-22.5)$

$18.9(13.3-24.4)$

$1.15(0.87-1.58)$

FH model

$19.8(19.2-20.5)$

$1.08(0.81-1.48)$

Women

FRP model

$13.6(13.0-14.2)$

$13.4(9.3-17.8)$

$1.04(0.76-1.45)$

FH model

$13.2(12.8-13.7)$

$1.01(0.75-1.42)$

${ }^{*} \mathrm{Cl}=$ confidence interval; FRP $=$ familial risk profile; $\mathrm{FH}=$ binary family history. 


\section{Figure Legends}

Figure 1: Cumulative incidence of colorectal cancer (CRC) according to estimated 5year absolute risk among a) population-based relatives, b) clinic-based relatives. Four groups were defined based on cut-points of $30^{\text {th }}, 60^{\text {th }}$ and $90^{\text {th }}$ percentiles of estimated 5-year absolute risk. The K-sample test was used to compare the cumulative incidence across groups and to calculate two-sided $P$ values. ${ }^{39}$ FRP $=$ familial risk profile

Figure 2. Age-adjusted Receiver Operating Characteristic (ROC) curves for men and women. ROC curves and Age-adjusted area under the curve (AUC) were calculated as the weighted average of age-specific estimates, with weights as the proportion of CRC diagnosis in each age group ( $<50$ and $>=50$ at baseline). We calculated $95 \%$ confidence intervals (in parentheses) using bootstrap approach. $\mathrm{FRP}=$ familial risk profile; $\mathrm{FH}=$ binary family history. 

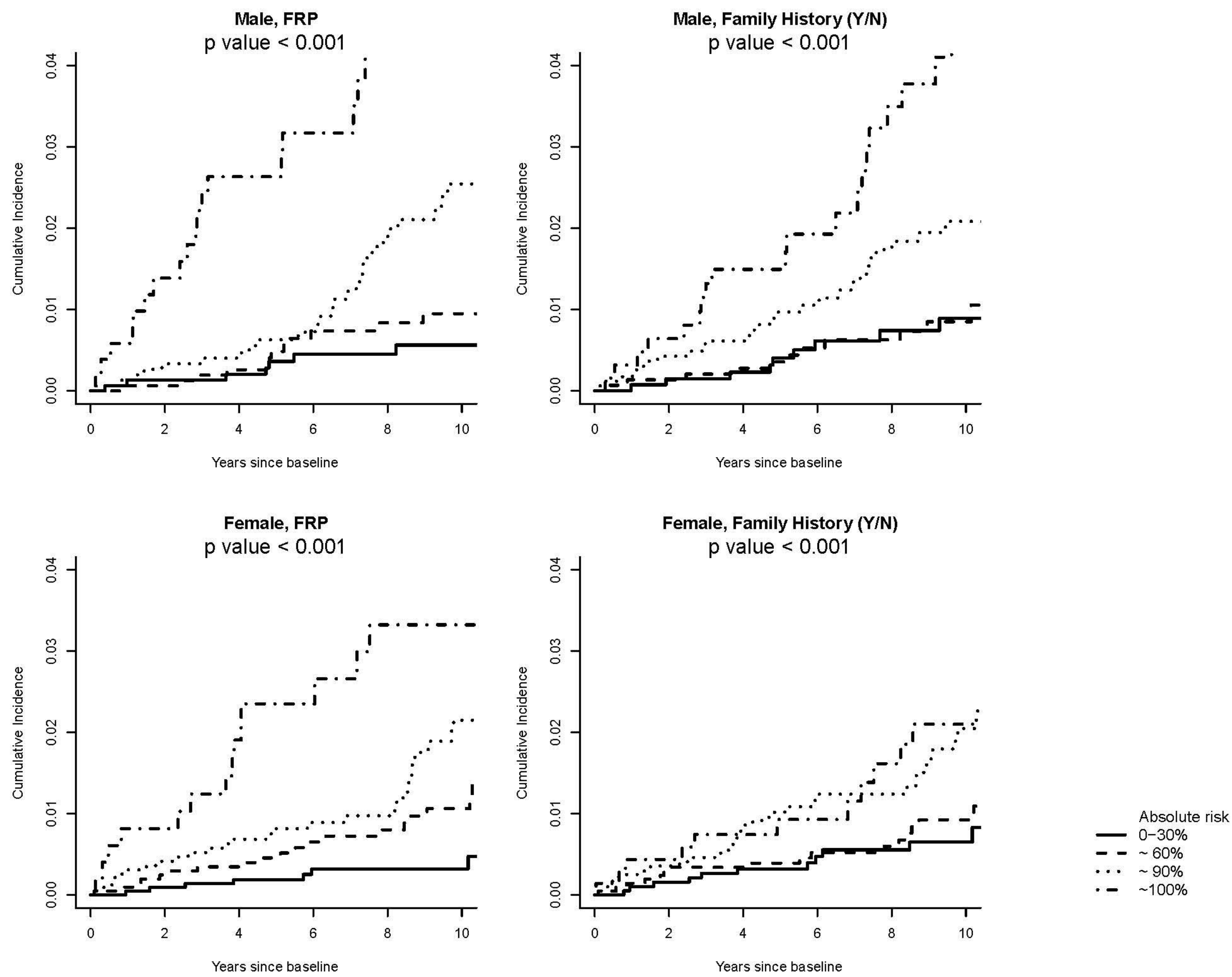

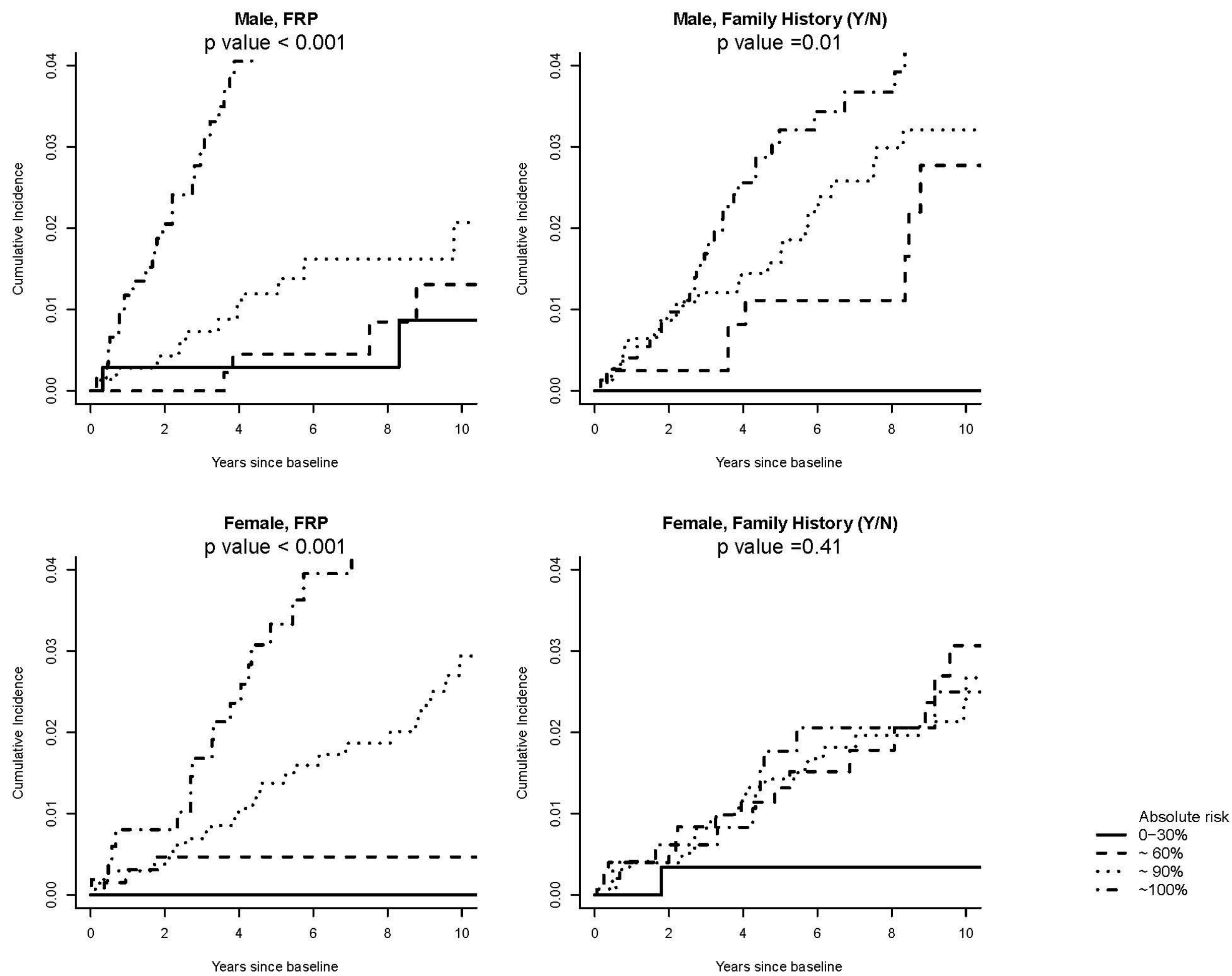
Population-based, Men

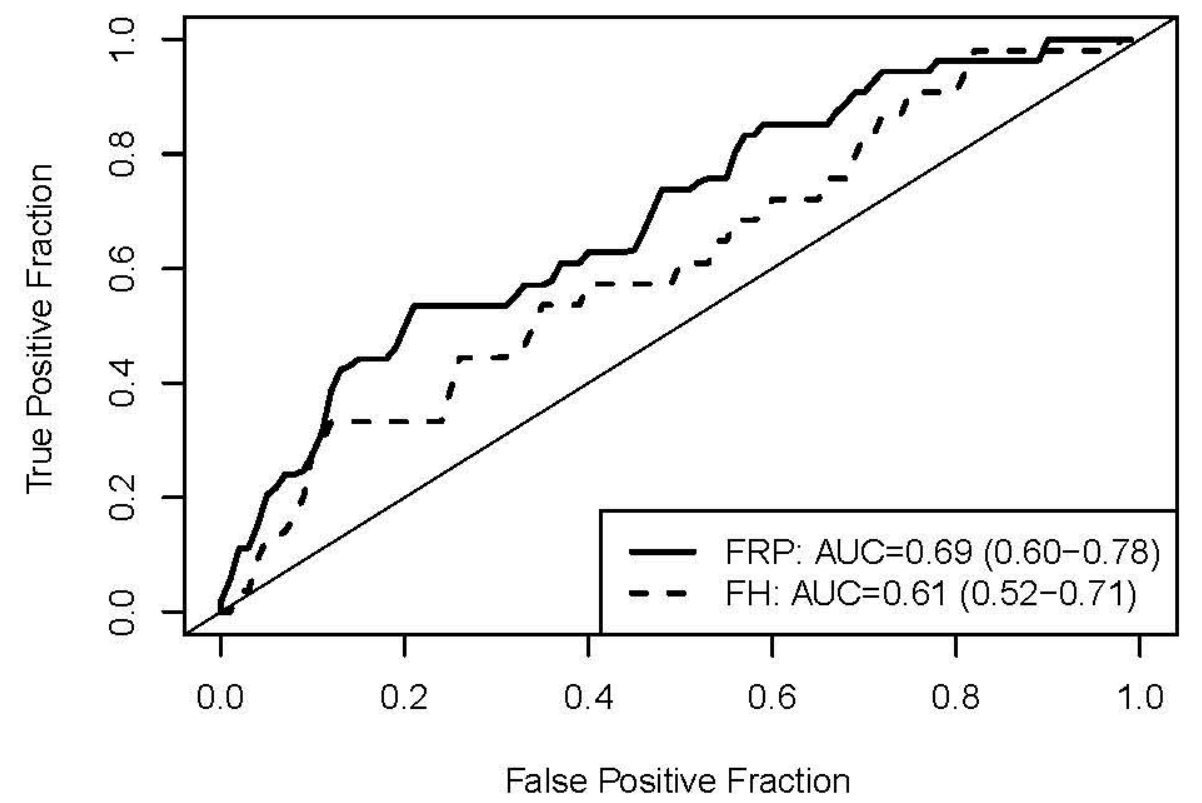

Clinic-based, Men

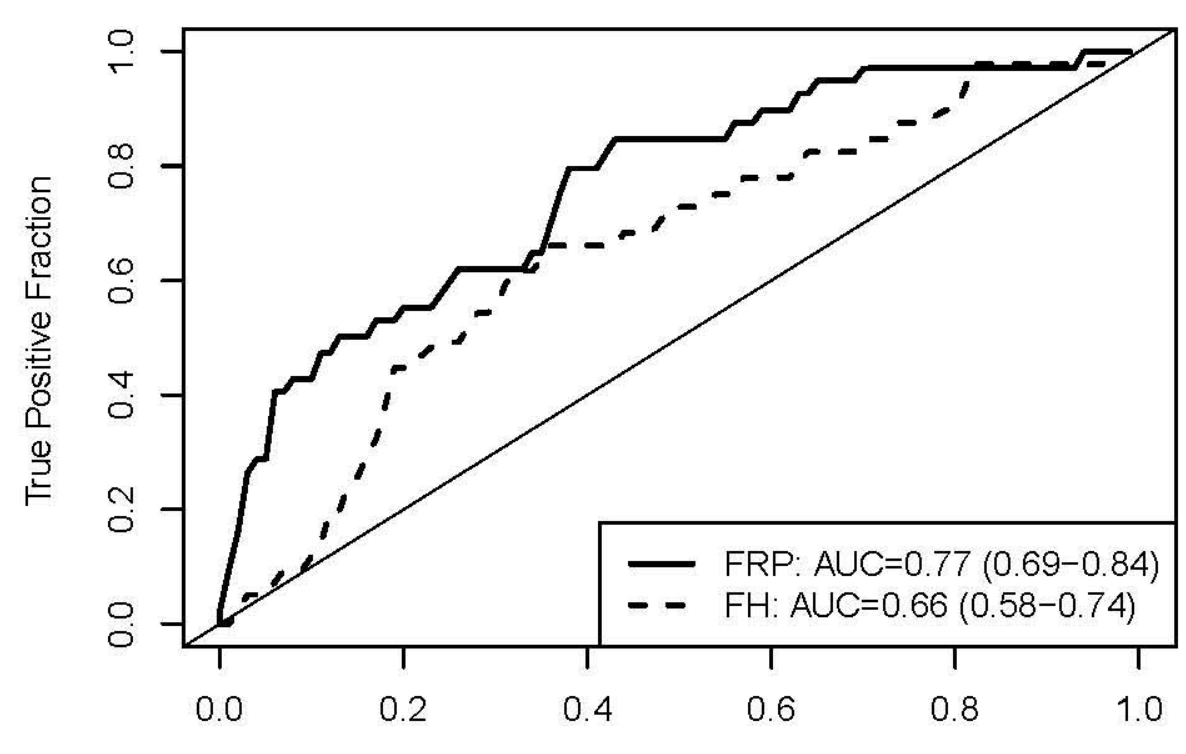

False Positive Fraction
Population-based, Women

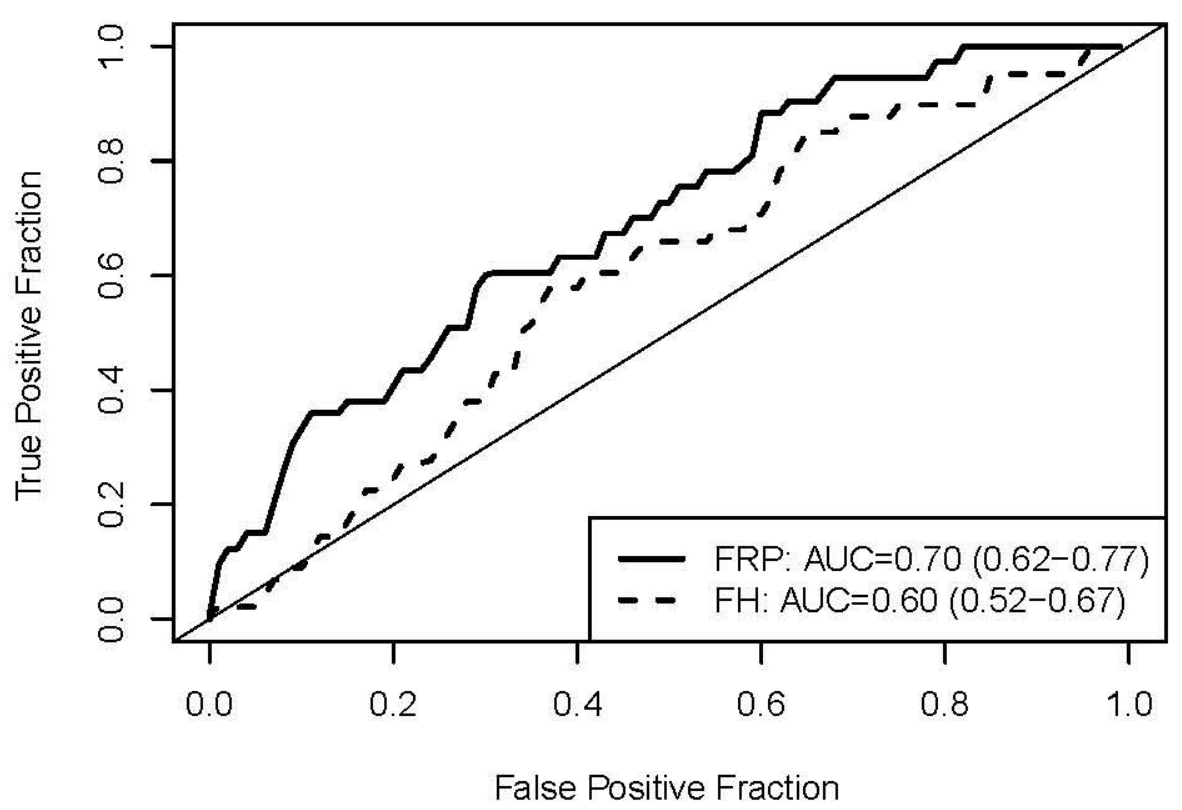

Clinic-based, Women

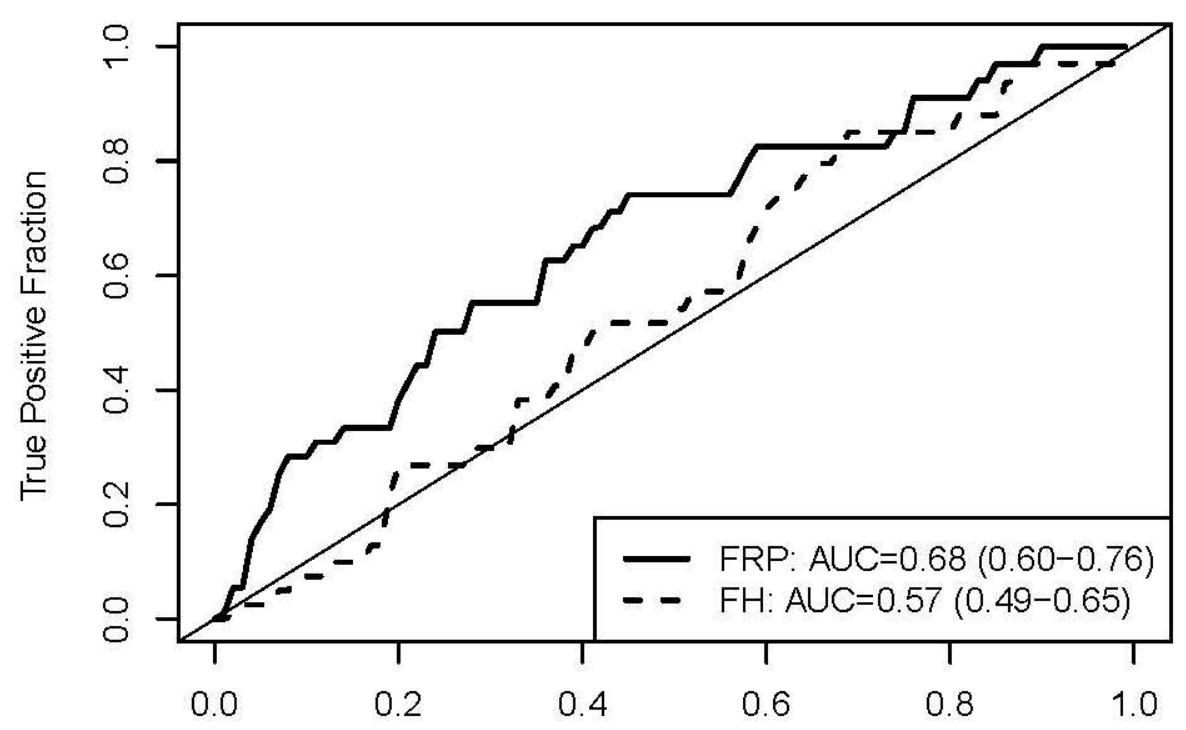

False Positive Fraction 